\title{
Stable potassium isotopic compositions of angrites: Implications for volatile element depletion in their parent body
}

\author{
YAN HU ${ }^{1}$ AND FRÉDÉRIC MOYNIER ${ }^{2}$ \\ ${ }^{1}$ IPGP CNRS UMR 7154 \\ ${ }^{2}$ Université de Paris, Institut de Physique du Globe de Paris, \\ CNRS
}

Presenting Author: yanhu@ipgp.fr

Terrestrial planets, the Moon, and most differentiated meteorites are depleted in volatile elements relative to the bulk solar composition. Constraining the mechanism and timing of the depletion remains challenging and is integral for reconstructing the accretion history and early evolution of the solar system. Potassium $(\mathrm{K})$ is a moderately volatile element, and $\mathrm{K} / \mathrm{U}$ ratio is widely used to assess the relative depletion of volatile elements in solar system materials. Recent analytical advances have motivated studies using stable $\mathrm{K}$ isotopes to trace planetary volatile histories. For example, lunar basalts and HED meteorites (howardite-eucrite-diogenite) are enriched in heavy $\mathrm{K}$ isotopes relative to chondrites and bulk silicate Earth, likely due to kinetic isotope fractionation during evaporation [1,2].

Angrites are rare meteorites that are the most alkali-depleted and among the oldest basaltic rocks in the solar system. Studies of these meteorites provide valuable insights into volatile element depletion in the early solar system [3]. However, the extremely low $\mathrm{K}$ concentrations in angrites make it difficult to measure their $\mathrm{K}$ isotopes accurately and precisely using conventional MC-ICPMS. With the advent of the collision cellequipped $\mathrm{Nu}$ Sapphire MC-ICPMS, K isotope measurements can be made on much smaller samples than previously possible [4]. Here we report the first high-precision dataset for angrites to decipher processes that depleted volatile element contents in their parent body. We found over $0.8 \%$ variation in $\delta^{41} \mathrm{~K}$, with all the values being substantially lower than major groups of chondrites. Such low values have not been documented in upper continental crust materials, and are also too low to reflect terrestrial contamination or weathering. The most likely scenario is that angrite parent body had lost the majority of its $\mathrm{K}$ inventory and a fraction of $\mathrm{K}$ recondensed kinetically, imparting the variably light $\mathrm{K}$ isotopic signatures to the angrites.

[1] Wang \& Jacobsen (2016), Nature 538, 487-490.

[2] Tian et al. (2019), GCA 266, 611-632.

[3] Pringle et al. (2014), PNAS 111, 17029-17032.

[4] Moynier et al. (2021), Chem. Geol., in press. 